\title{
Das Selbststudium der Studierenden
}

\author{
Ergebnisse einer Befragung zur zeitlichen und räumlichen \\ Organisation des Lernens
}

\section{Einleitung}

$\mathrm{Zu}$ einem Studium gehört nicht nur der Besuch von Lehrveranstaltungen, sondern auch das selbstorganisierte Lernen. Mit der Einführung der Bachelor- und Masterstudiengänge ist der komplette Workload der Studierenden (Lehrveranstaltungen plus Selbststudium) in den Fokus der Studienanforderungen gerückt. Hinzu kommen neue kombinierte Lehr- und Lernformen wie etwa das projektorientierte Lernen. Die Hochschulen benötigen daher nicht nur eine bedarfsgerechte Ausstattung an Lehrräumen (Hörsäle, Seminarräume etc.), sondern auch ein passendes Angebot an studentischen Arbeitsplätzen für das Selbststudium.

Unter dem Begriff „Selbststudium“ werden im Folgenden alle im Zusammenhang mit dem Studium erforderlichen Tätigkeiten außerhalb des Besuchs von Lehrveranstaltungen zusammengefasst: beispielsweise das Vor- und Nachbereiten von Lehrveranstaltungen, die Anfertigung schriftlicher Arbeiten, die Ausleihe und Lektüre von Fachliteratur oder die Prüfungsvorbereitungen.

Bereits 2013 wurde vom HIS-Institut für Hochschulentwicklung (HIS-HE) in Kooperation mit dem Deutschen Zentrum für Hochschul- und Wissenschaftsforschung (DZHW) eine bundesweite empirische Online-Befragung durchgeführt. Ziel war es, Daten darüber zusammenzutragen und auszuwerten, an welchen Orten und mit welchem zeitlichen Aufwand die Studierenden ihr Selbststudium verbringen und welche Rolle hierbei speziell die Hochschulbibliotheken spielen. Diese Befragung wurde 2018, also eine Generation von Studierenden später, wiederholt. Der vorliegende Beitrag fasst die zentralen Ergebnisse der Studie zusammen. Zunächst wird der zeitliche Aufwand für das Studium und für das Lernen dargelegt. Daran an schließt sich die Analyse, an welchen Orten die Studierenden lernen.

Dieser Beitrag verfolgt primär das Ziel, die Ergebnisse der Befragung deskriptiv darzulegen, um die Diskussionen über das Thema Selbststudium und 
über den Bedarf an Arbeitsplätzen für das selbstorganisierte Lernen der Studierenden in der Hochschule zu versachlichen. ${ }^{1}$

\section{Präferenzen der Studierenden}

Die Studierenden wurden zu Beginn danach gefragt, welche Präferenzen sie grundsätzlich bei der Wahl ihres Lernortes haben: „In Situationen, in denen Sie den Ort des Selbststudiums frei wählen können: Für welchen Ort entscheiden sie sich vorzugsweise?“. Im Ergebnis zeigt sich, das rund zwei Drittel der Studierenden am liebsten zu Hause lernen, rund ein Drittel dagegen in der Hochschule. An Fachhochschulen wird weniger in der Hochschule gelernt als an Universitäten. Insgesamt lassen sich vier Präferenztypen identifizieren (Abbildung 1).

\section{Bevorzugter Ort des Selbststudiums (Angaben in\%)}

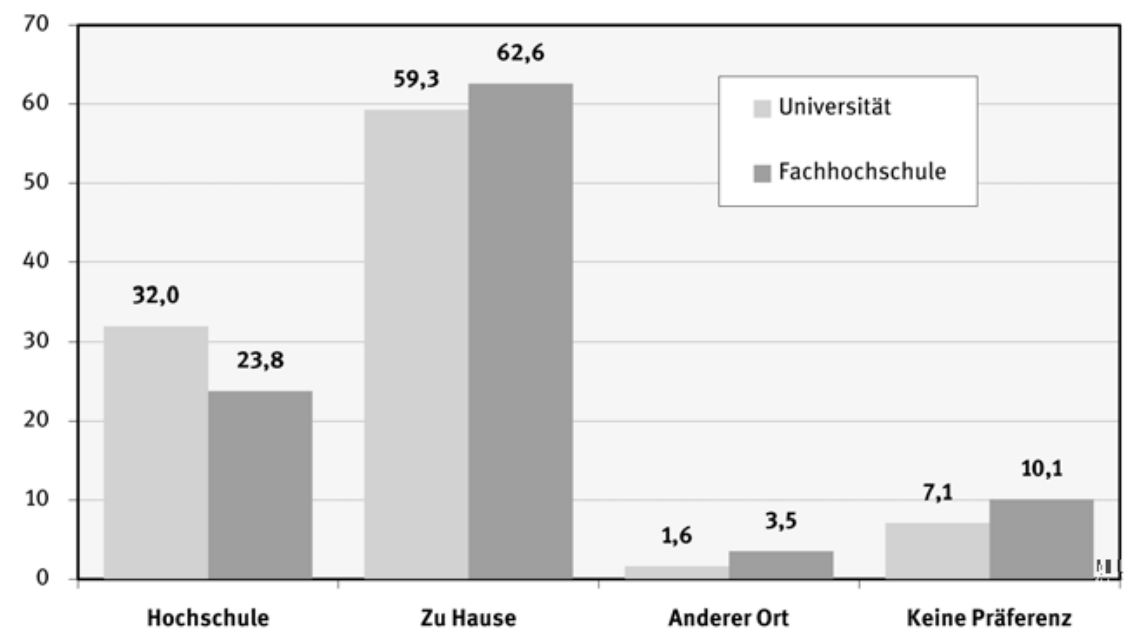

Abb. 1: Bevorzugter Ort des Selbststudiums (eigene Darstellung).

Die Bevorzugung des Homeoffice vor dem Lernen in der Hochschule zeigte sich bereits in der letzten Studie von 2013 und gilt für Universitäten wie Fachhochschulen und für alle Fächergruppen. Allerdings gibt es von Fach zu Fach deutliche Unterschiede: Bei den Fächergruppen Recht und Medizin finden sich die

1 Die komplette Studie „Orte des Selbststudiums 2018“ findet sich unter: https://his-he.de/publikationen/detail/publikation/orte-des-selbststudiums-2018/. 
höchsten Werte für das Lernen in der Hochschule (40\%), der niedrigste Wert entfällt auf Lehramtsstudiengänge (16\%).

Als wichtigste Gründe für das Selbststudium zu Hause werden genannt:

- flexible Zeiteinteilung,

- Ruhe,

- Verpflegungsmöglichkeiten.

Folgende Gründe sprechen aus Sicht der Studierenden für das Lernen in der Hochschule:

- Kontakt mit Kommilitoninnen und Kommilitonen,

- Fachliteratur vor Ort,

- fachlicher Austausch.

$\mathrm{Zu}$ Hause wird vor allem dann gelernt, wenn es auf Ruhe und flexible Einteilung der Lernzeiten ankommt. Das Lernen in der Hochschule steht dann im Vordergrund, wenn kommunikative Aspekte im Mittelpunkt stehen.

Die Befragung zeigt auch, dass die Studierenden in der großen Mehrheit mit den aktuell vorhandenen Bedingungen zum Selbststudium in der Hochschule zufrieden sind (Abbildung 2).

Zufriedenheit mit den Möglichkeiten zum Selbststudium an der Hochschule (Angaben in \%)

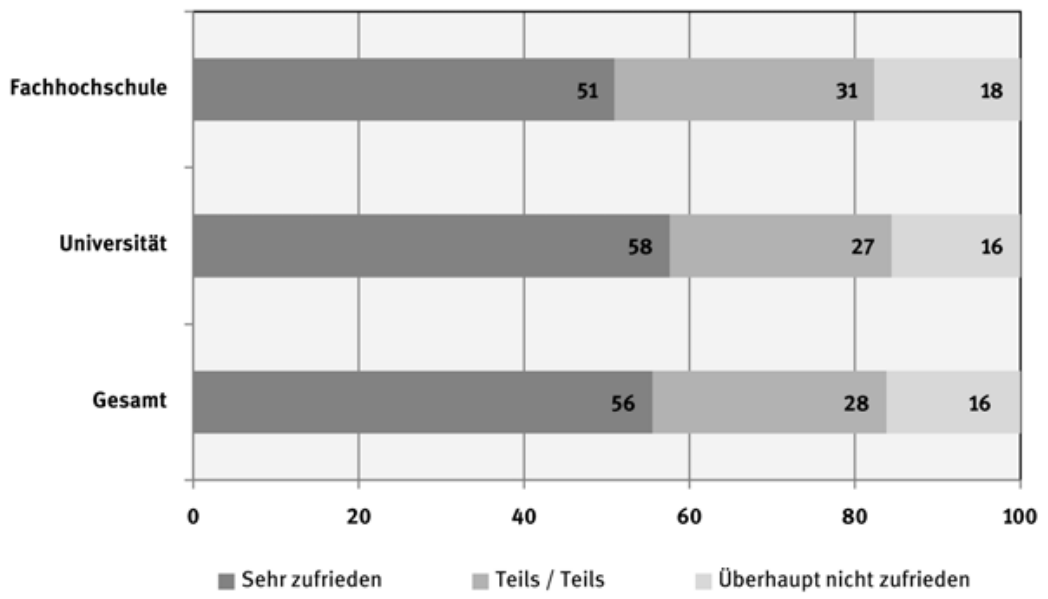

Abb. 2: Zufriedenheit mit den Möglichkeiten des Selbststudiums an der Hochschule (eigene Darstellung).

Lediglich 16 bis 18 Prozent der Studierenden sind mit den Möglichkeiten für das Selbststudium in der Hochschule überhaupt nicht zufrieden. 


\section{Zeitbudget: Studium gesamt}

Die Auswertung der Befragung „Orte des Selbststudiums 2018“ hat für das Wintersemester 2017/2018 zunächst den Gesamtumfang des studienbezogenen Zeitaufwands erhoben. Der Gesamtaufwand der Studierenden für Lehrveranstaltungen und Selbststudium beträgt im Mittel 30,9 Stunden (plus 6,0 Stunden Verteilzeit). Im Vergleich zur Befragung von 2013 bedeutet dies einen Rückgang des Zeitaufwandes für Lehrveranstaltungen um rund 4,0 Stunden/Woche. Alle übrigen Aufwände sind etwa konstant. Dieser Rückgang des Zeitaufwandes der Studierenden für Lehrveranstaltungen korrespondiert mit den Ergebnissen der 21. Sozialerhebung des Deutschen Studentenwerks (Middendorf et al. 2017). Zu den Gründen für diesen signifikanten Rückgang des Zeitaufwandes für Lehrveranstaltungen können keine Aussagen getroffen werden.

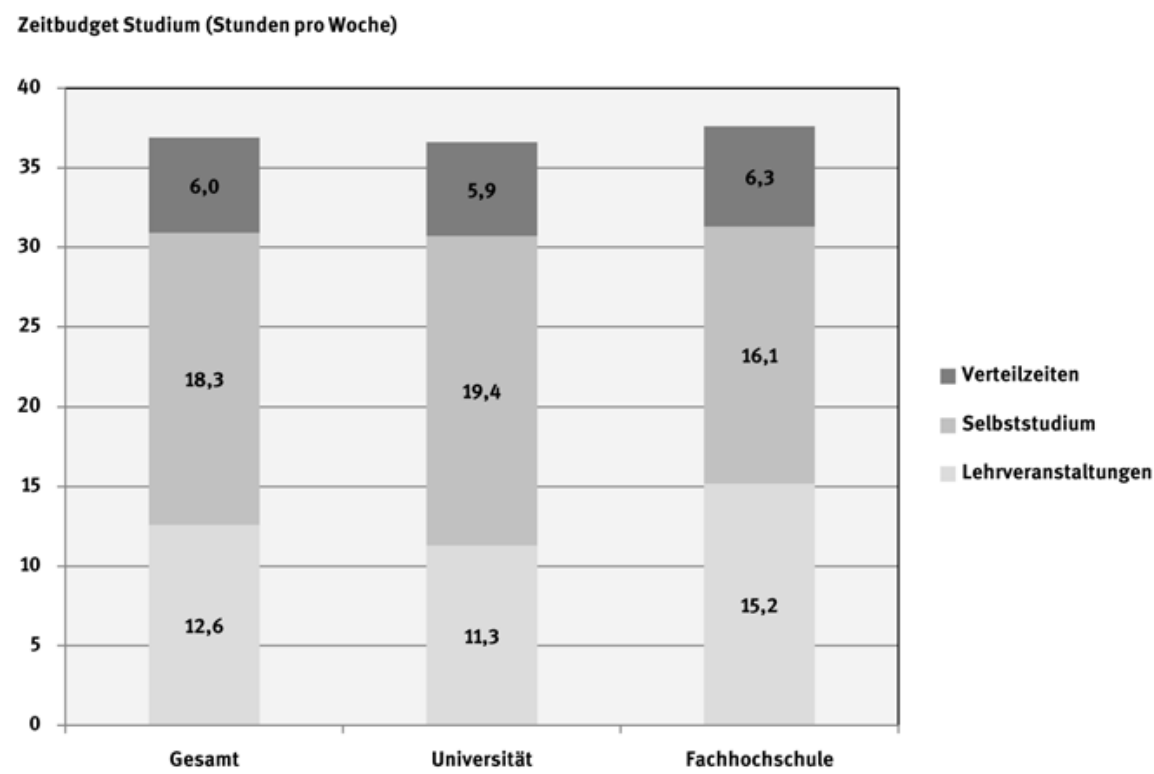

Abb. 3: Zeitbudget Studium (eigene Darstellung).

Auf die Lehrveranstaltungen entfallen den Angaben der Studierenden zufolge 12,6 Stunden, auf das Selbststudium 18,3 Stunden (Abbildung 3). Zusätzlich wurden die Verteilzeiten abgefragt (Anfahrt, Wartezeiten etc.), die im Mittel bei rund 6 Stunden pro Woche liegen, so dass sich ein Gesamtaufwand von knapp 37 Wochenstunden ergibt. 
Der zeitliche Aufwand für Lehrveranstaltungen und Selbststudium ist von Fächergruppe zu Fächergruppe sehr unterschiedlich. Die aktuelle Erhebung weist eine Streuung des zeitlichen Aufwandes für das Studium von 41,7 Stunden/ Woche für Studierende der Medizin an Universitäten bis 26,0 Stunden für Studierende der Rechtswissenschaften an Fachhochschulen aus. An Universitäten ist der Aufwand für das Selbststudium deutlich höher als für Lehrveranstaltungen.

\section{Zeitbudget: Selbststudium}

Aufschlussreich ist zunächst die Differenzierung danach, ob das Selbststudium grundsätzlich zu Hause oder in der Hochschule stattfindet. Insgesamt betrachtet werden die rund 19 Stunden Selbststudium zu rund 60 Prozent zu Hause und zu rund 40 Prozent in der Hochschule verbracht. Die Verteilung des Selbststudiums auf häusliche Tätigkeit oder Zeiten in der Hochschule variiert nur wenig im Vergleich zwischen Universitäten und Fachhochschulen. Allerdings liegt der absolute Zeitaufwand für das Selbststudium bei den Studierenden der Fachhochschulen sowohl in der Hochschule als auch zu Hause im Mittel rund 2,5 Stunden unter dem Aufwand der Studierenden an Universitäten (Abbildung 4).

\section{Zeitbudget Selbststudium (Stunden pro Woche)}

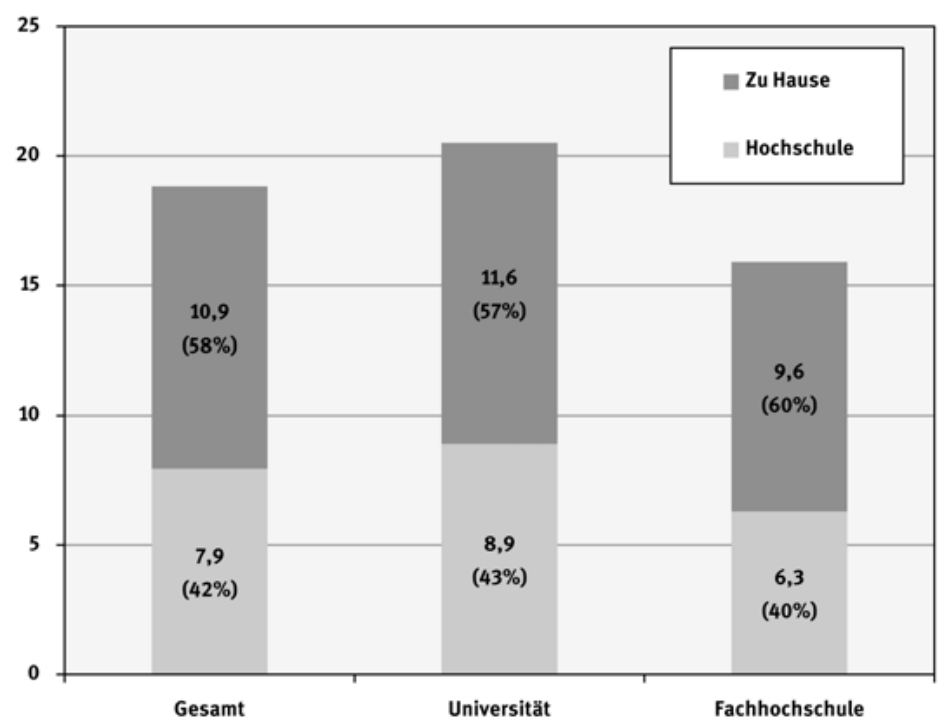

Abb. 4: Zeitbudget Selbststudium (eigene Darstellung). 
Zeitbudget Selbststudium Universität (Stunden/Woche)

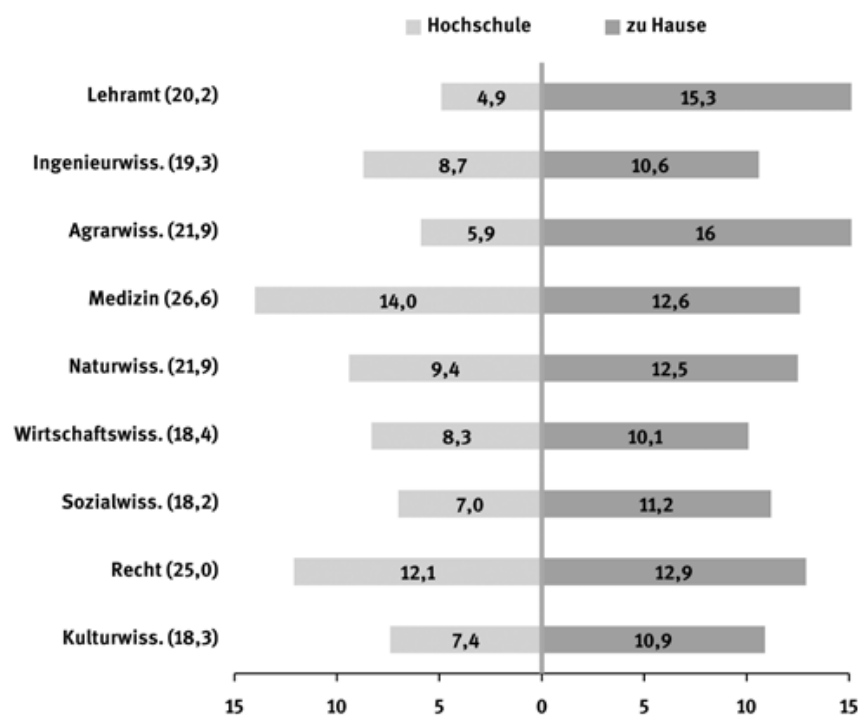

Abb. 5: Zeitbudget Selbststudium Universität (eigene Darstellung).

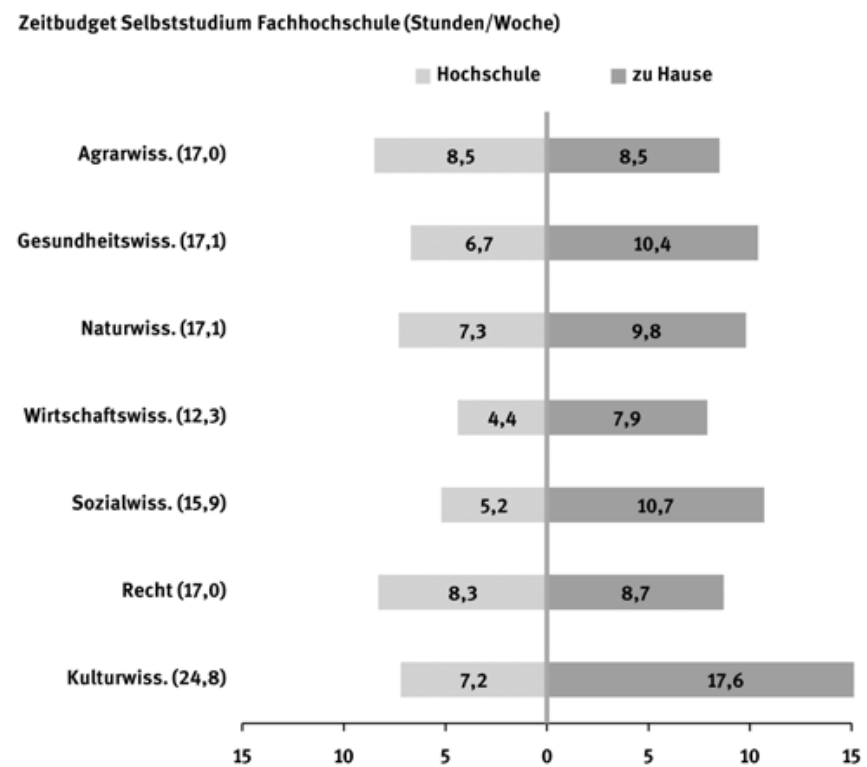

Abb. 6: Zeitbudget Selbststudium Fachhochschule (eigene Darstellung). 
Betrachtet man die Verteilung des Selbststudiums auf die Orte „zu Hause“ und „Hochschule“ differenziert nach Fächergruppen, so zeigen sich deutliche fächerspezifische Unterschiede (Abbildungen 5 und 6).

Die Bandbreite des zeitlichen Aufwands für das Selbststudium reicht an Universitäten von 18,2 Stunden/Woche in den Sozialwissenschaften bis zu 26,6 Stunden/Woche in der Medizin. Für das Lernen in der Hochschule wenden die Studierenden der Medizin und der Rechtswissenschaften die meiste Zeit auf. An den Fachhochschulen sind die Zeitbudgets für das Lernen zu Hause oder in der Hochschule signifikant niedriger und betragen zwischen 4,4 und 8,5 Stunden/ Woche.

Die Betrachtung der Mittelwerte vermittelt nur ein unzureichendes Bild der tatsächlichen Situation des Lernens: Hinter den Mittelwerten verbirgt sich eine erhebliche empirische Streuung: Ein großer Teil der befragten Studierenden erbringt nur einen geringen oder sogar keinen Aufwand für das Selbststudium. Ein kleiner Teil der Studierenden dagegen erbringt sehr hohe zeitliche Aufwände, so dass von einer starken Polarisierung der Studierenden gesprochen werden kann. Der Blick auf die Streuung des Zeitaufwandes für das Selbststudium in der Hochschule verdeutlicht diese Situation (Abbildung 7).

\section{Selbststudium in der Hochschule Zeitaufwand pro Woche (Angaben in \%)}

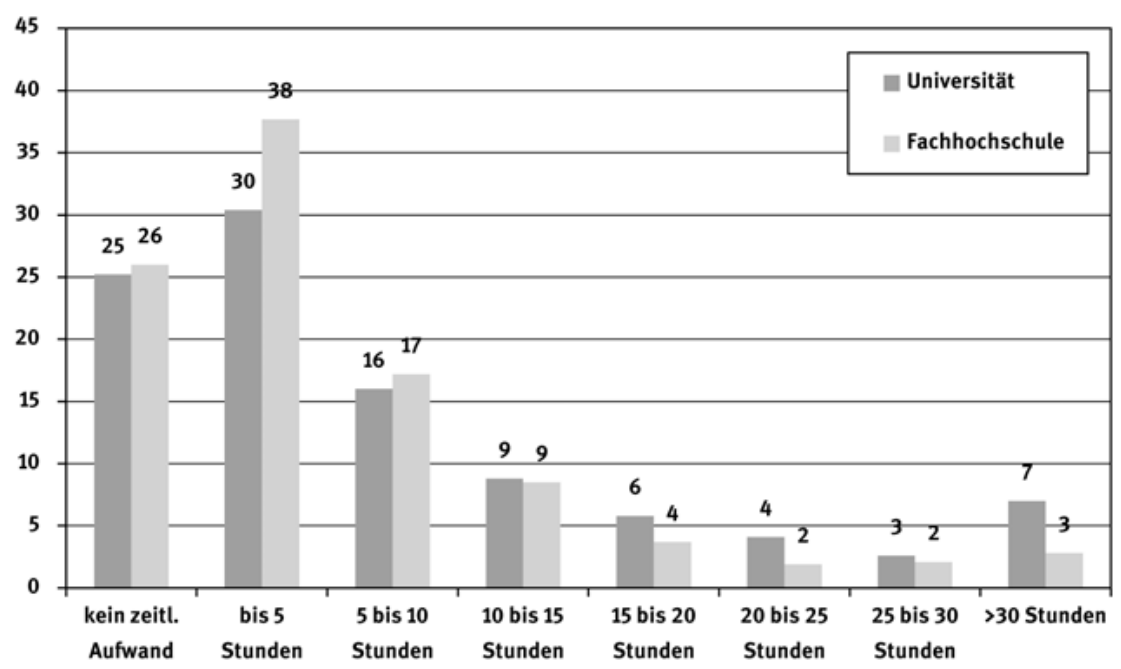

Abb. 7: Selbststudium in der Hochschule - Zeitaufwand (eigene Darstellung). 
Auf der einen Seite lernen 55 Prozent (Universität) beziehungsweise 64 Prozent (Fachhochschule) der Studierenden maximal 5 Stunden/Woche in der Hochschule; auf der anderen Seite der Skala lernen 14 Prozent (Universität) beziehungsweise 7 Prozent (Fachhochschule) mehr als 20 Stunden/Woche in der Hochschule. Nur eine kleine Gruppe von etwa 16-17 Prozent der Studierenden erbringt einen Aufwand, der dem Mittelwert entspricht. Eine ähnliche Verteilung zeigt sich, wenn man auf die zeitliche Streuung des Zeitbudgets für das Lernen zu Hause schaut.

Dieser wichtige Befund verdeutlicht, dass der Bedarf an Arbeitsplätzen für das Selbststudium in der Hochschule vor allem von einer kleinen Gruppe von Studierenden generiert wird, die intensiv in der Hochschule lernen. Wenn man die Grenze bei 10 Stunden/Woche Selbststudium in der Hochschule zieht, dann beträgt der Anteil dieser Gruppe von „Intensivnutzerinnen und -nutzern“ an Universitäten rund 30 Prozent, an Fachhochschulen rund 20 Prozent.

\section{Orte des Selbststudiums in der Hochschule}

Für die Hochschulen ist nicht nur von Bedeutung, in welchem zeitlichen Umfang die Studierenden in der Hochschule lernen, sondern auch, welche Räumlichkeiten hierfür aufgesucht und welche Arten von Arbeitsplätzen genutzt werden.

Zeitbudget Selbststudium in der Hochschule (Stunden/Woche) nach Lernorten

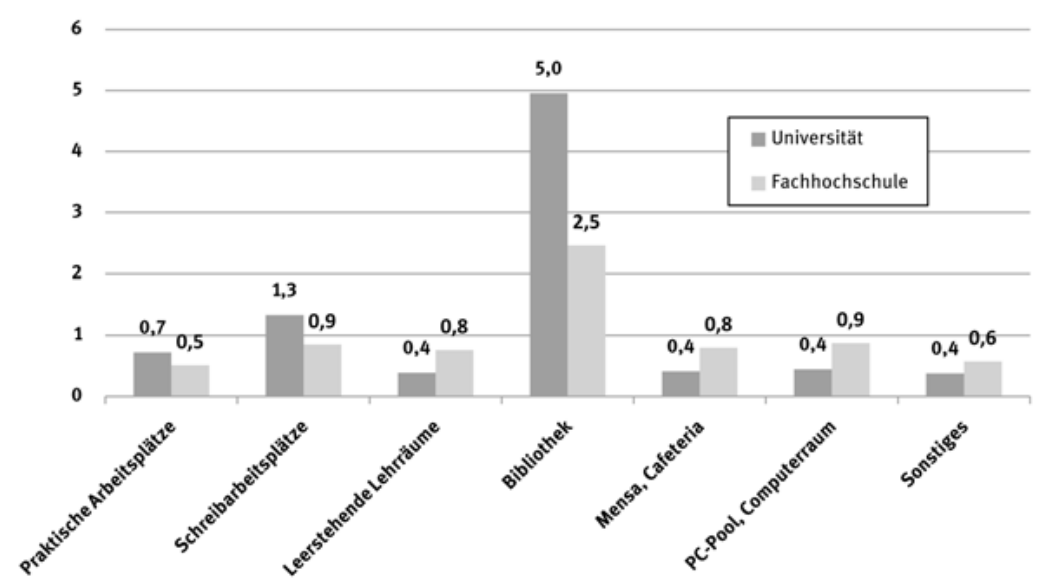

Abb. 8: Selbststudium in der Hochschule - Zeitbudget nach Lernorten (eigene Darstellung). 
Wie oben dargelegt, beträgt der mittlere zeitliche Aufwand für das Selbststudium in der Hochschule 8,9 Stunden/Woche an Universitäten und 6,3 Stunden/ Woche an Fachhochschulen. Dieses Zeitbudget verteilt sich auf die wichtigsten Orte des Selbststudiums in der Hochschule wie in Abbildung $8 \mathrm{zu}$ sehen ist.

Ein Großteil des Zeitbudgets für das Selbststudium wird in den Bibliotheken abgedeckt: Durchschnittlich 5,0 Stunden/Woche verbringen die Studierenden an Universitäten in der jeweiligen Hochschulbibliothek. Demgegenüber ist an Fachhochschulen der Stellenwert der Bibliotheken deutlich geringer, der zeitliche Aufwand liegt im Mittel nur bei 2,5 Stunden/Woche. Den übrigen möglichen Orten des Selbststudiums kommt gegenüber der Bibliothek eine relativ geringe Bedeutung zu. Dies ist natürlich auch darauf zurückzuführen, dass gegenwärtig die meisten Plätze für das Selbststudium in den Bibliotheken angeboten werden. Dieser generelle Befund hat sich gegenüber der Studie von 2013 nicht verändert.

Zwischen den Fächergruppen variiert der Stellenwert der einzelnen Orte des Selbststudiums in der Hochschule erheblich. Den unterschiedlichen Stellenwert der einzelnen Lernorte verdeutlicht beispielhaft die Analyse des Lernverhaltens von Studierenden der Naturwissenschaften. In dieser Fächergruppe ist die Fachkultur des Lehrens und Lernens stark durch experimentelle Studienanteile geprägt. Die Studierenden der Naturwissenschaften müssen große Anteile ihres Studiums in Lehrlaboren (Praktikumsräumen) und den angrenzenden Auswerteplätzen verbringen. Die Hochschulbibliotheken spielen eine deutlich geringere Rolle als bei den „klassischen“ Buchwissenschaften (Abbildung 9).

\section{Zeitbudget Selbststudium in der Hochschule (Stunden/Woche) nach Lernorten: Fächergruppe Naturwissenschaften (Universităt)}

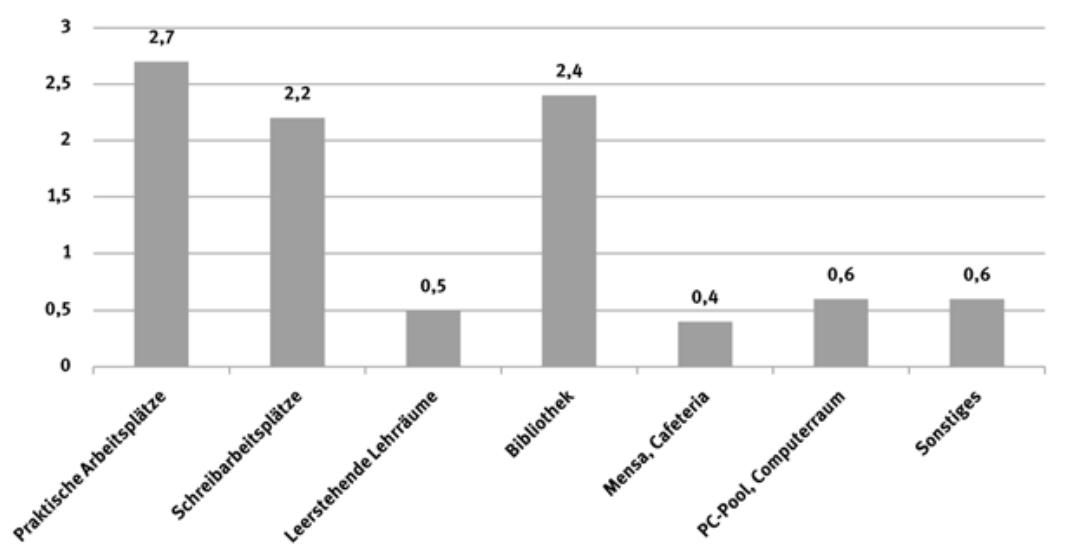

Abb. 9: Zeitbudget Selbststudium nach Lernorten in der Fächergruppe Naturwissenschaften (eigene Darstellung). 
Studierende der Naturwissenschaften lernen durchschnittlich nur 2,4 Stunden/ Woche in der Hochschulbibliothek, dagegen werden praktisch-experimentelle Arbeitsplätze und Schreib-/Auswerteplätze in der Nähe der Labore in annähernd gleichem Umfang genutzt. Alle übrigen möglichen Lernorte spielen nur eine untergeordnete Rolle.

\section{Nutzung von Hochschulbibliotheken}

Wie oben dargelegt, beträgt der mittlere Zeitaufwand für das Lernen in der $B i$ bliothek 5,0 Stunden an Universitäten beziehungsweise 2,5 Stunden an Fachhochschulen. Bei der Betrachtung des Gesamtaufwandes für das Selbststudium zeigt sich, dass eine starke Polarisierung des Zeitbudgets $\mathrm{zu}$ beobachten ist: Über die Hälfte der Studierenden lernt wenig oder gar nicht in der Hochschule, nur 14 Prozent an Universitäten beziehungsweise 7 Prozent an Fachhochschulen lernen mehr als 20 Stunden/Woche in der Hochschule. Dieses heterogene Bild wiederholt sich beim zeitlichen Aufwand für das Lernen speziell in der Hochschulbibliothek (Abbildung 10).

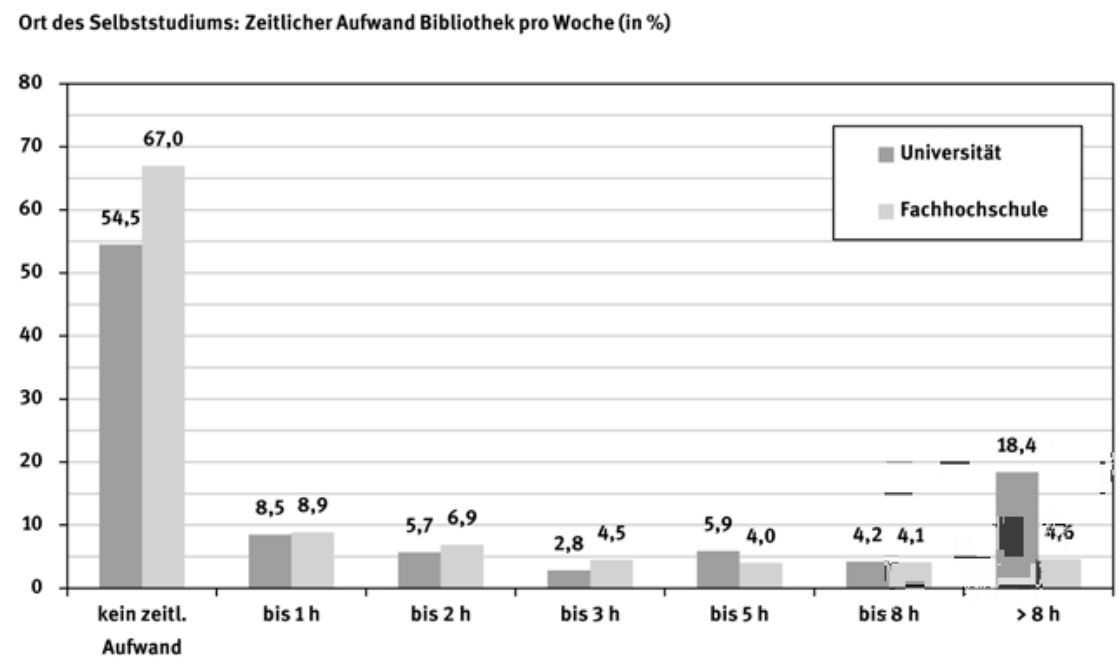

Abb. 10: Zeitlicher Aufwand zum Selbststudium in der Bibliothek (eigene Darstellung).

An den Universitäten verbringen fast 70 Prozent der Studierenden maximal 2 Stunden/Woche in der Bibliothek, an den Fachhochschulen sind es sogar über 80 Prozent. Die Nutzungsdauer von bis zu einer Stunde legt die Vermutung 
nahe, dass überwiegend Bücher ausgeliehen oder abgegeben werden, Literatur recherchiert oder Texte kopiert werden; ein kontinuierliches Selbststudium an den Arbeitsplätzen der Bibliothek findet dagegen aufgrund des geringen Zeitbudgets nicht statt. Am anderen Ende des Spektrums lernen rund 18 Prozent (Universität) beziehungsweise 5 Prozent (Fachhochschule) mehr als 8 Stunden in der Hochschulbibliothek. Studierende, deren wöchentliches Zeitbudget um den jeweiligen Mittelwert herum liegt, sind dagegen deutlich in der Minderheit. Insgesamt zeigt sich, dass die meisten Studierenden kaum oder gar nicht in der Bibliothek lernen, ein kleiner Anteil dagegen sehr intensiv seine Lernzeit in der Bibliothek verbringt.

Diesem generellen Befund entsprechen auch die Antworten auf die Frage nach den überwiegenden Tätigkeiten in der Bibliothek. 23 Prozent der Studierenden an Fachhochschulen und 39 Prozent der Studierenden an Universitäten geben an, während der Vorlesungszeit an den vorhandenen Arbeitsplätzen in den Bibliotheken zu lernen. Diese Angaben haben sich im Vergleich zur Studie von 2013 erhöht.

Demgegenüber geben rund 47 Prozent der Studierenden an Universitäten und rund 62 Prozent der Studierenden an Fachhochschulen an, die Bibliotheken überwiegend für die Ausleihe von Büchern, für die Literaturrecherche und für Kopiertätigkeiten zu nutzen. Für die Mehrheit der Studierenden ist die Bibliothek offensichtlich primär kein Ort des Lernens, sondern ein Ort, um sich Materialien für das Lernen zu Hause zu beschaffen (Abbildung 11).

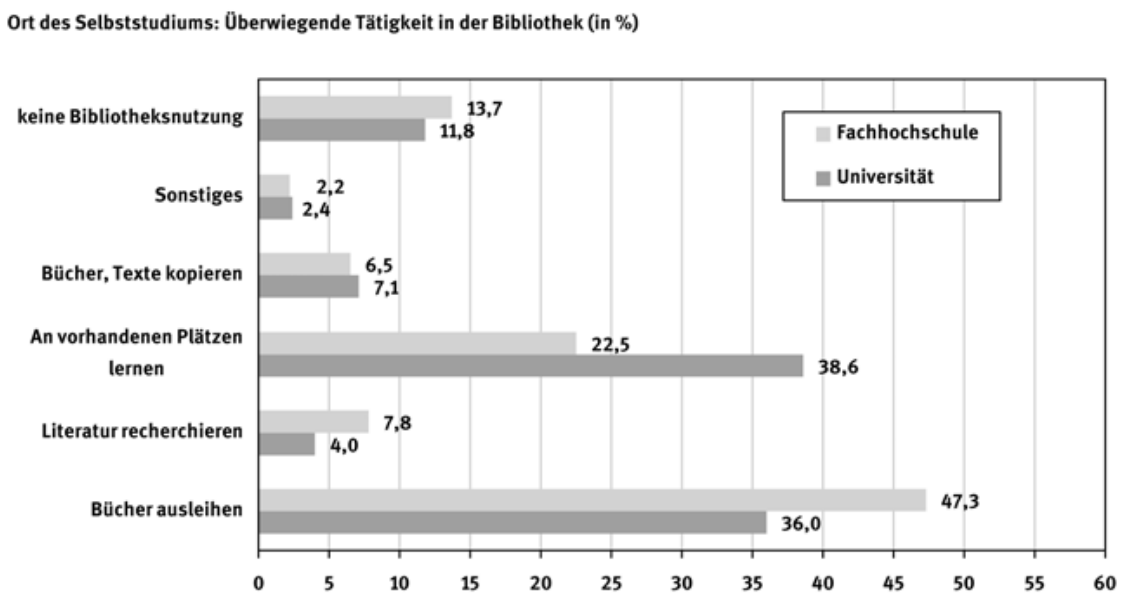

Abb. 11: Überwiegende Tätigkeit beim Selbststudium in der Bibliothek (eigene Darstellung). 
Ort des Selbststudiums: Zeitlicher Aufwand Bibliothek pro Woche (in \%): Recht

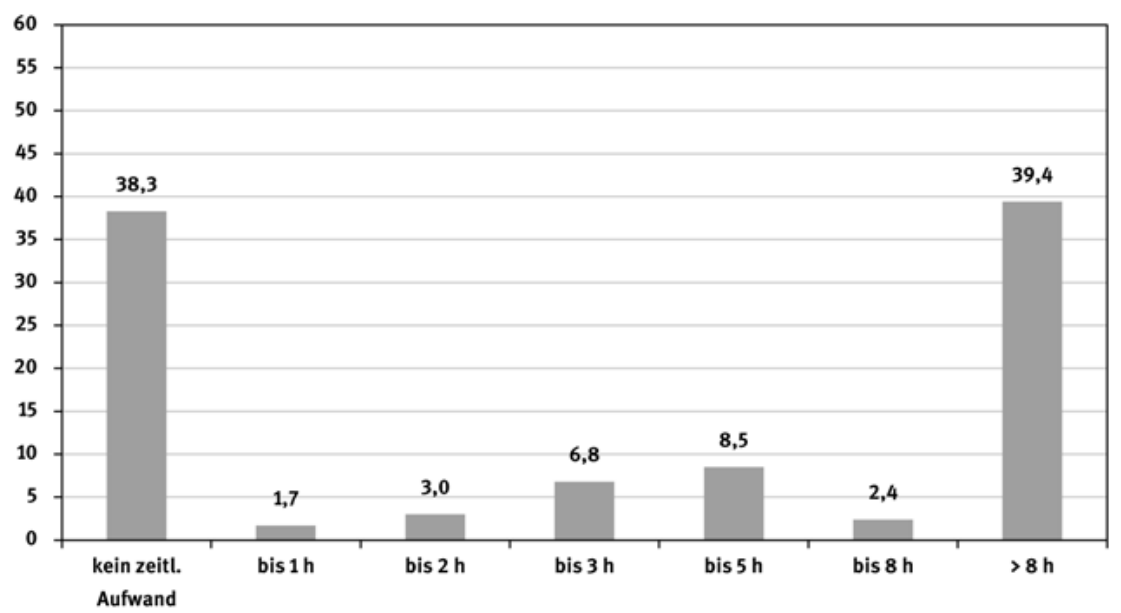

Abb. 12: Zeitlicher Aufwand zum Selbststudium in der Bibliothek im Bereich Recht (eigene Darstellung).

Ort des Selbststudiums: Zeitlicher Aufwand Bibliothek pro Woche (in \%): Naturwissenschaften

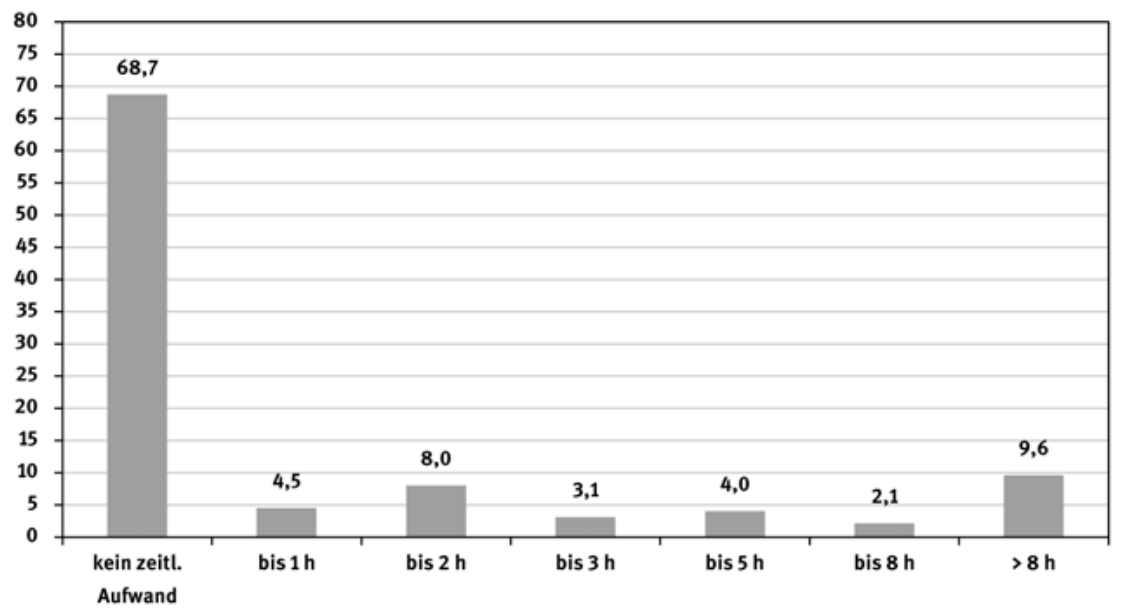

Abb. 13: Zeitlicher Aufwand zum Selbststudium in der Bibliothek im Bereich Naturwissenschaften (eigene Darstellung). 
Das gespaltene Verhältnis der Studierenden zum Lernen in der Hochschule zeigt sich auch bei einer differenzierten Betrachtung nach Fachkulturen. Um die Unterschiede herauszuarbeiten, zeigen die Abbildungen 12 und 13 den zeitlichen Aufwand, den Studierenden der Rechtswissenschaften und der Naturwissenschaften in der Bibliothek erbringen.

Bei den Jura-Studierenden ist der Anteil, der mehr als 8 Stunden/Woche in der Bibliothek lernt, mit 40 Prozent wesentlich höher als der mittlere Aufwand aller Studierenden. Trotzdem zeigt sich auch hier eine deutliche Polarisierung: eine fast ebenso große Gruppe erbringt keinen zeitlichen Aufwand in der Bibliothek.

Bei der Fächergruppe der Naturwissenschaften dagegen ist die Bedeutung der Hochschulbibliotheken als Lernort erheblich geringer als der Durchschnitt: fast 70 Prozent der Studierenden erbringen keinen zeitlichen Aufwand in der Bibliothek, lediglich 10 Prozent lernen über 8 Stunden/Woche in der Hochschulbibliothek.

Seit Jahren wird darüber spekuliert, ob die zunehmenden Online-Angebote der Hochschulbibliotheken Auswirkungen auf die Nutzung der Bibliotheksgebäude haben. Neu in die Befragung aufgenommen wurde daher der Aspekt, ob die neuen digitalen Angebote der Hochschulbibliotheken durch die Studierenden angenommen werden und an welchem Lernort diese Angebote genutzt werden.

Bei der Frage nach der Nutzung digitaler Angebote der Hochschulbibliotheken gibt die große Mehrheit der Studierenden an, dass diese Angebote umfangreich genutzt werden, und zwar 82 Prozent an Universitäten und 76 Prozent an Fachhochschulen (Abbildung 14).

\section{Nutzung digitaler Angebote der Bibliothek (in \%)}

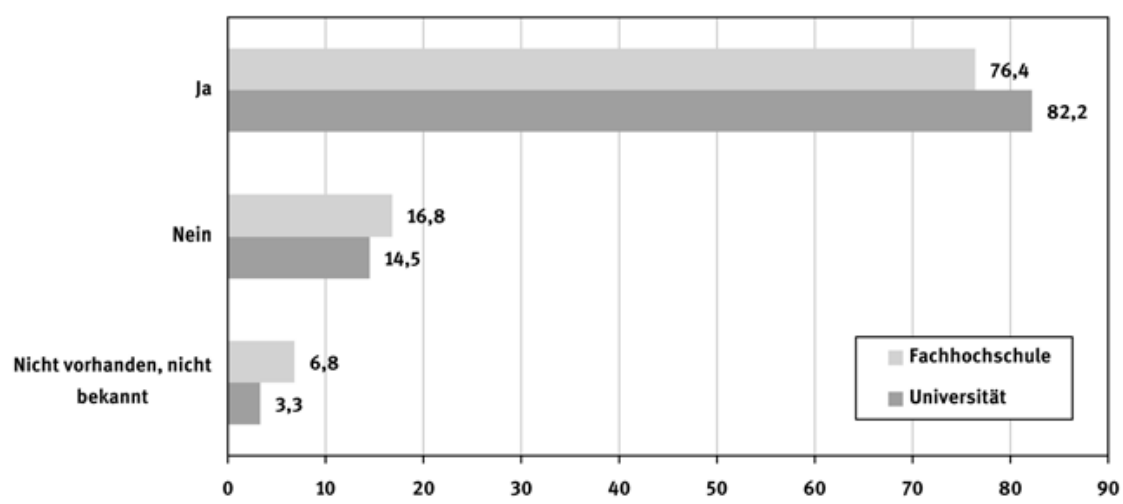

Abb. 14: Nutzung digitaler Angebote der Bibliothek (eigene Darstellung). 
Welche Angebote werden genutzt? Sowohl an Universitäten als auch an Fachhochschulen werden E-Books und der OPAC am meisten genutzt. Über die Hälfte der Studierenden nutzen diese digitalen Angebote sehr häufig. Sehr selten werden E-Learning-Angebote genutzt (Abbildungen 15 und 16).

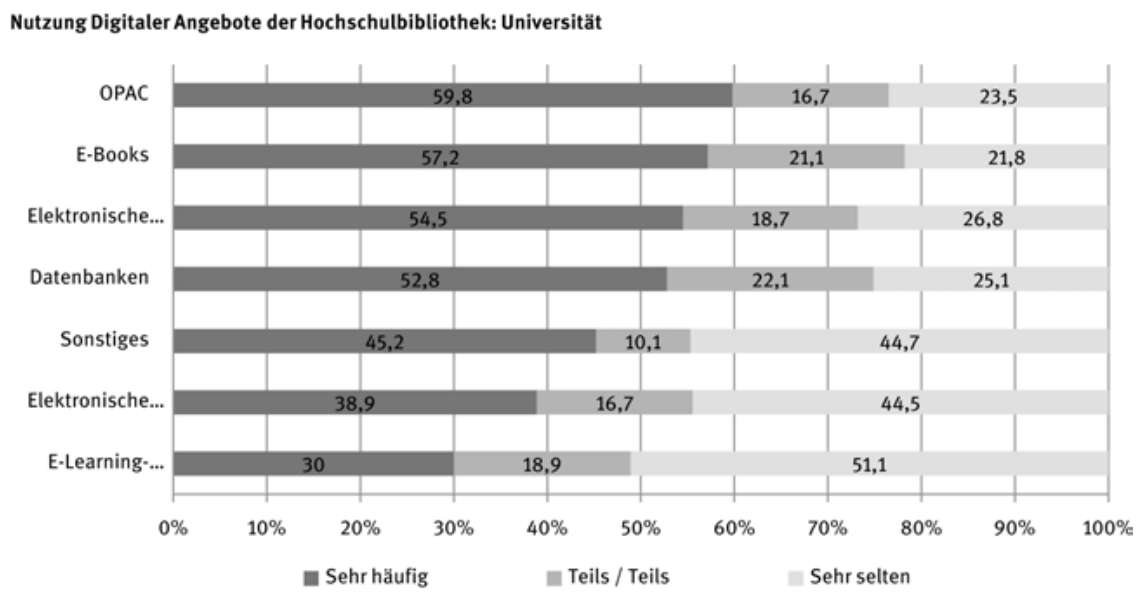

Abb. 15: Nutzung digitaler Angebote der Bibliothek in Universitäten (eigene Darstellung).

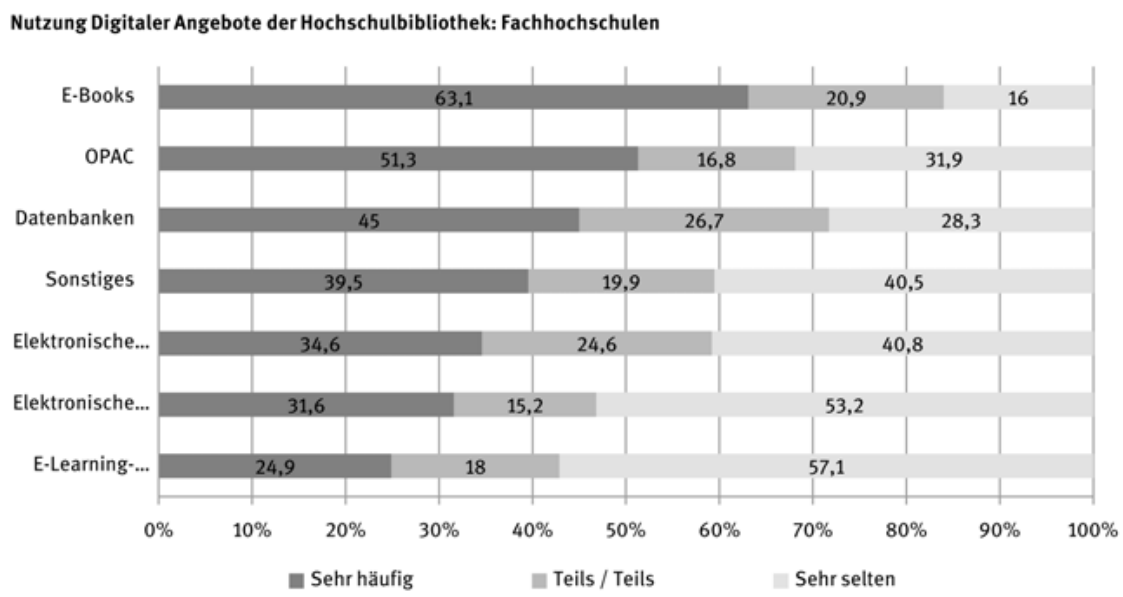

Abb. 16: Nutzung digitaler Angebote der Bibliothek in Fachhochschulen (eigene Darstellung). 
Wo werden die digitalen Angebote der Hochschulbibliotheken von den Studierenden genutzt? Die Möglichkeit, bibliothekarische Dienstleistungen ubiquitär nutzen zu können, ohne das Bibliotheksgebäude betreten zu müssen, wird von vielen Studierenden im Studienalltag umgesetzt: 67 Prozent (Universität) beziehungsweise 77 Prozent (Fachhochschule) der Studierenden nutzen die digitalen Angebote von zu Hause aus (Abbildung 17).

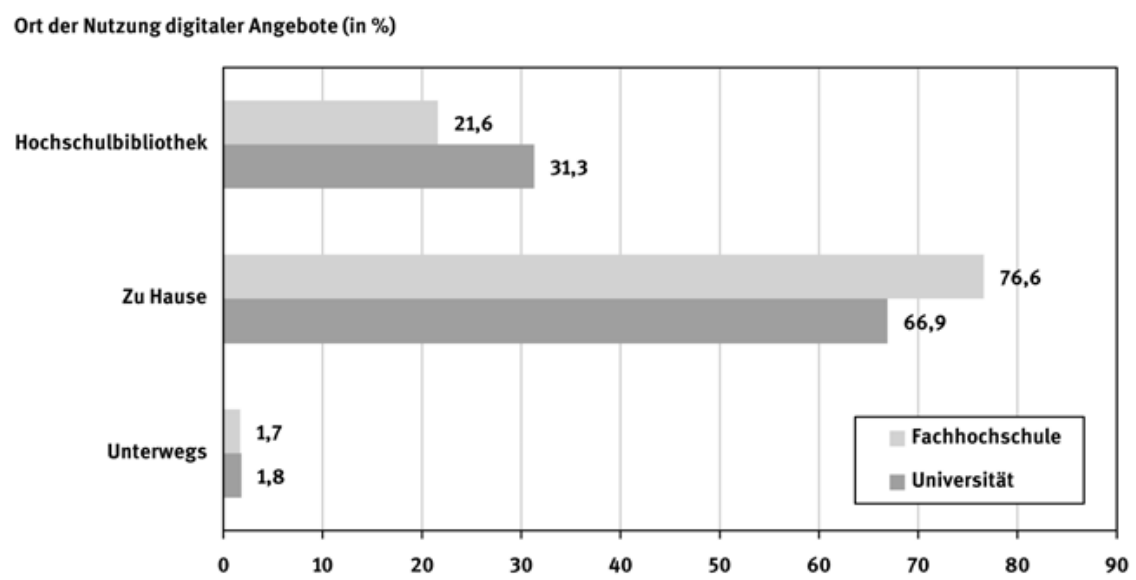

Abb. 17: Orte der Nutzung digitaler Angebote (eigene Darstellung).

\section{Fazit}

Wie sieht die zukünftige Gestaltung von physischen, digitalen und hybriden Lernräumen an den Hochschulen aus? Neue Lehrmethoden und veränderte didaktische Konzepte sollten auf die unterschiedlichen Bedürfnisse der Studierenden reagieren und die verschiedenen Fachkulturen berücksichtigen. Die Lernwelt Hochschule gibt es nur im Plural.

Grundsätzlich kann festgehalten werden, dass derzeit der überwiegende Teil des Selbststudiums zu Hause stattfindet. Begründet wird dies von den Studierenden mit den Arbeitsbedingungen und der Flexibilität der Arbeitsorganisation, aber auch mit der Verpflegungssituation zu Hause.

Sowohl der Blick auf die Zeitbudgets als auch auf die gewählten Orte des Selbststudiums zeigt, dass sich die Studierenden bei der zeitlichen und räumlichen Organisation ihres Selbststudiums deutlich unterscheiden. Insbesondere ist die Polarisierung zwischen zwei Gruppen von Studierenden hervorzuheben: Am einen Ende der Bandbreite eine große Gruppe von Studierenden, die nur 
wenig Zeit in das Selbststudium insgesamt und das Lernen in der Hochschule speziell investieren; auf der anderen Seite eine kleine Gruppe von Studierenden, die sehr zeitintensiv lernen. Diese Gruppe generiert den Bedarf an studentischen Arbeitsplätzen für das Selbststudium

Die Wahl der Lernorte hängt letztlich von einer ganzen Reihe von Einflussfaktoren ab (Abbildung 18).

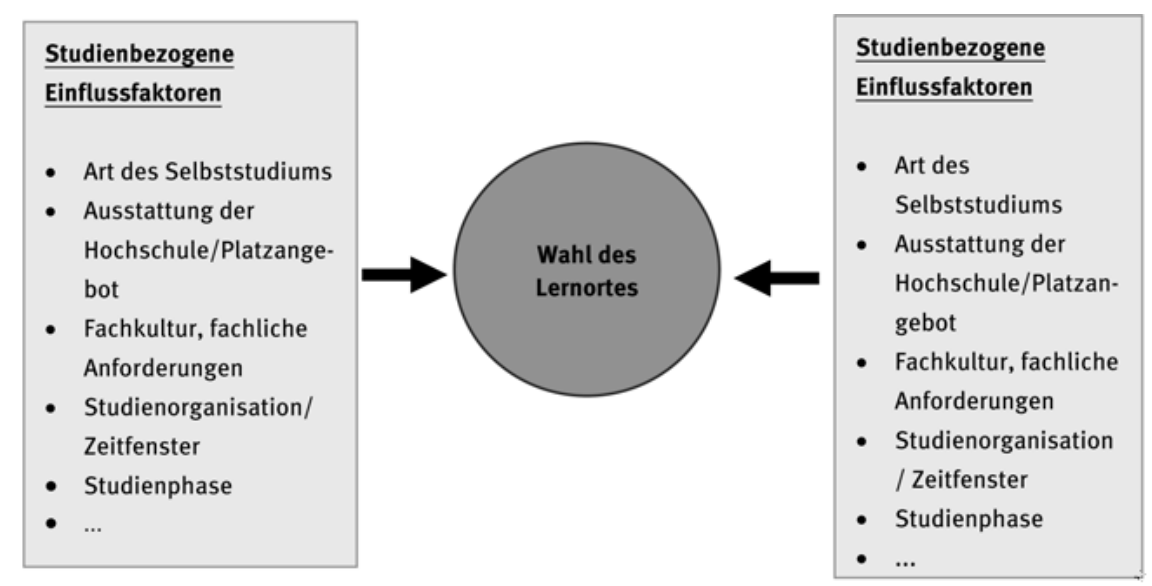

Abb. 18: Faktoren bei der Wahl des Lernorts (eigene Darstellung).

Zum einen handelt es sich um Einflussfaktoren, die im Studium/in den Studienbedingungen selbst zu verorten sind (studienbezogene Einflussfaktoren), zum anderen lassen sich auch in den persönlichen Rahmenbedingungen der Studierenden Einflussgrößen finden (individuelle Einflussfaktoren).

Aus der Perspektive der Hochschulforschung liefern die Befunde zu den Zeitbudgets und den Präferenzen beim Selbststudium sowie das hier dargestellte Modell der Einflussfaktoren auf die Wahl der Lernorte einen guten Überblick darüber, wie Studierende ihr Selbststudium organisieren. Die Studie verweist aber zugleich unmittelbar auf weiteren Forschungsbedarf: Aufgrund des Erhebungsdesigns können nur Aussagen für die Vorlesungszeit getroffen werden. Inwieweit sich in der vorlesungsfreien Zeit oder in speziellen Prüfungsphasen im Semester Umfang und Organisation des Selbststudiums verändern, insbesondere mit Blick auf die Wahl des Lernortes, muss somit im Rahmen dieser Untersuchung offenbleiben. Des Weiteren stellt sich die Frage, wie sich die hier vorgestellten Befunde im Zeitverlauf (weiter-)entwickeln werden, insbesondere vor dem Hintergrund studienstruktureller Veränderungen und des steigenden Angebotes an alternativen Lehr- und Lernorten. 